Research Article

\title{
Hepatoprotective Effect of Polysaccharides Isolated from Dendrobium officinale against Acetaminophen-Induced Liver Injury in Mice via Regulation of the Nrf2-Keap1 Signaling Pathway
}

\author{
Guosheng Lin $\mathbb{D}^{1},{ }^{1,2}$ Dandan Luo, ${ }^{1,2}$ Jingjing Liu, ${ }^{1,2}$ Xiaoli Wu $\mathbb{D}^{3},{ }^{3}$ Jinfen Chen, ${ }^{1,2}$ \\ Qionghui Huang, ${ }^{1,2}$ Lingye Su, ${ }^{4}$ Lei Zeng, ${ }^{4}$ Hongfeng Wang $\left(\mathbb{0},{ }^{4}\right.$ and Ziren Su $\mathbb{C}^{1,2}$ \\ ${ }^{1}$ Mathematical Engineering Academy of Chinese Medicine, Guangzhou University of Chinese Medicine, Guangzhou 510006, China \\ ${ }^{2}$ Guangdong Provincial Key Laboratory of New Drug Development and Research of Chinese Medicine, Guangzhou University of \\ Chinese Medicine, Guangzhou 510006, China \\ ${ }^{3}$ Postdoctoral Programme, Department of Second Institute of Clinical Medicine, Guangzhou University of Chinese Medicine, \\ Guangzhou 510006, China \\ ${ }^{4}$ Guangdong Provincial Key Laboratory of Silviculture, Protection and Utilization, Guangdong Academy of Forestry, \\ Guangzhou 510520, China
}

Correspondence should be addressed to Hongfeng Wang; wanghf@sinogaf.cn and Ziren Su; suziren@126.com

Received 27 January 2018; Revised 20 April 2018; Accepted 20 May 2018; Published 18 July 2018

Academic Editor: Walid Rachidi

Copyright ( 2018 Guosheng Lin et al. This is an open access article distributed under the Creative Commons Attribution License, which permits unrestricted use, distribution, and reproduction in any medium, provided the original work is properly cited.

The effect of polysaccharides isolated from Dendrobium officinale (DOP) on acetaminophen- (APAP-) induced hepatotoxicity and the underlying mechanisms involved are investigated. Male Institute of Cancer Research (ICR) mice were randomly assigned to six groups: (1) control, (2) vehicle (APAP, $230 \mathrm{mg} / \mathrm{kg}$ ), (3) $N$-acetylcysteine (100 mg/kg), (4) $50 \mathrm{mg} / \mathrm{kg}$ DOP, (5) $100 \mathrm{mg} / \mathrm{kg}$ DOP, and (6) $200 \mathrm{mg} / \mathrm{kg}$ DOP. Alanine aminotransferase (ALT) and aspartate aminotransferase (AST) levels in the serum and glutathione (GSH), malondialdehyde (MDA), catalase (CAT), total antioxidant capacity (T-AOC), myeloperoxidase (MPO), and reactive oxygen species (ROS) levels in the liver were determined after the death of the mice. The histological examination of the liver was also performed. The effect of DOP on the Kelch-like ECH-associated protein 1- (Keap1-) nuclear factor erythroid 2-related factor 2 (Nrf2) signaling pathway was evaluated using Western blot analysis and real-time polymerase chain reaction (PCR). The results showed that DOP treatment significantly alleviated the hepatic injury. The decrease in ALT and AST levels in the serum and ROS, MDA, and MPO contents in the liver, as well as the increases in GSH, CAT, and T-AOC in the liver, were observed after DOP treatment. DOP treatment significantly induced the dissociation of Nrf2 from the Nrf2-Keap1 complex and promoted the Nrf2 nuclear translocation. Subsequently, DOP-mediated Nrf2 activation triggered the transcription and expressions of the glutamate-cysteine ligase catalytic (GCLC) subunit, glutamate-cysteine ligase regulatory subunit (GCLM), heme oxygenase-1 (HO-1), and $\mathrm{NAD}(\mathrm{P}) \mathrm{H}$ dehydrogenase quinone 1 (NQO1) in APAP-treated mice. The present study revealed that DOP treatment exerted potentially hepatoprotective effects against APAP-induced liver injury. Further investigation about mechanisms indicated that DOP exerted the hepatoprotective effect by suppressing the oxidative stress and activating the Nrf2-Keap1 signaling pathway.

\section{Introduction}

Drug-induced liver injury (DILI) has become one of the most frequent causes leading to cessation of drug test in clinical trials, restrictions on drug use, and withdrawal of approved drugs [1]. The overall occurrence of DILI is between $0.00001 \%$ and $0.01 \%$ patient-years $[2,3]$. DILI can broadly be divided into predictable and dose-dependent, such as 
acetaminophen (paracetamol, APAP), and unpredictable or idiosyncratic DILI (IDILI). IDILI is a significant health problem due to its unpredictability, potential cause for mortality, and poorly understood pathogenesis $[4,5]$. APAP overdose accounts for $50 \%$ of the acute liver failure cases in a predictable, dose-dependent, and intrinsically hepatotoxic manner $[6,7]$. Moreover, nearly half of the liver transplantation cases caused by DILI in the United States are attributed to APAP alone or in combination with other drugs [8]. APAP is an over-the-counter drug widely used as antipyretic and analgesic [9]. APAP is safe at therapeutic doses, but it is easy to be overused due to the individual difference. Furthermore, liver injury induced by APAP overdose occurs quickly within 24$48 \mathrm{~h}$ after ingestion [10]. At present, the clinical treatment for APAP-induced hepatotoxicity is extremely limited. $\mathrm{N}$-Acetylcysteine (NAC) is the most effective first-line antidote [11], but its effectiveness is restricted to the early stages of APAP-induced toxicity [12]. Hence, more effective and safe drugs to relieve the liver injury induced by APAP need to be urgently developed.

Recently, herbal products have attracted great attention as a major part of alternative medicine $[13,14]$. A large population chooses naturally derived medicines to maintain health and treat diseases [15]. Companies are also making attempts to discover new drugs of plant origin and perform structural modifications. Herbal drugs promote the regeneration of liver cells and accelerate the healing process in liver disorders [16].

Dendrobium officinale Kimura et Migo, called "Tie-PiShi-Hu" in Chinese, is an original food material and medicinal plant. As a traditional Chinese medicine in China, D. officinale is generally used to reinforce stomach function and generate body fluid [17]. Modern pharmacological studies indicate that $D$. officinale displays immunomodulatory, antidiabetic, anti-inflammatory, anticancer, hepatoprotective, and antioxidative activities [18, 19]. Chemical composition studies reveal that $D$. officinale mainly consists of polysaccharides, alkaloids, amino acids, and trace elements $[18,19]$. Among these chemical ingredients, polysaccharides isolated from $D$. officinale have an active role in food and health in human diet [20]. In particular, polysaccharides extracted from D. huoshanense, another species of Dendrobium, displayed hepatoprotective activities against ethanol- and carbon tetrachlorideinduced liver injuries [21, 22]. However, the effect and the mechanism of action of polysaccharides isolated from D. officinale (DOP) against APAP-induced liver injury remain unknown.

The present study is aimed at investigating the hepatoprotective effects of DOP against APAP-induced liver injury in mice. Specifically, the levels of alanine aminotransferase (ALT) and aspartate aminotransferase (AST) in serum were measured to assess the liver injury. Glutathione (GSH), malondialdehyde (MDA), reactive oxygen species (ROS), and myeloperoxidase (MPO) levels in the liver were determined to demonstrate oxidative damage. Catalase (CAT) and total antioxidant capacity (T-AOC) were also examined to test the antioxidative capacity. Also, the underlying mechanisms of the Nrf2-Keap1 signaling pathway were investigated using Western blot analysis and real-time polymerase chain reaction (PCR).

\section{Materials and Methods}

2.1. Chemicals and Reagents. D. officinale was kindly supplied by the Guangdong Academy of Forestry (Guangzhou, China). APAP was purchased from Shanghai Macklin Biochemical Co., Ltd. (Shanghai, China). NAC was obtained from Guangzhou Feibo Biological Technology Co., Ltd. (Guangzhou, China). Biochemical assay kits of ALT (C0092), AST (C0010-2), GSH (A006-1), MDA (A003-1), MPO (A004), CAT (A007-1), and T-AOC (A015) were purchased from Nanjing Jiancheng Bioengineering Institute (Nanjing, China). The mouse tissue ROS enzyme-linked immunosorbent assay (ELISA) kit (E-20634) was bought from Beijing Cheng Lin Biological Technology Co., Ltd. (Beijing, China). Nrf2 (sc-365949) and Keap1 (sc-365626) antibodies were obtained from Santa Cruz Biotechnology Inc. (CA, USA). Histone H3 (E021130-03), $\beta$-actin (E021020-03), and secondary antibodies (E030110-01) were provided by EarthOx, LLC (CA, USA). Primers of glutamate-cysteine ligase catalytic subunit (GCLC), glutamate-cysteine ligase regulatory subunit (GCLM), heme oxygenase-1 (HO-1), NAD(P)H dehydrogenase quinone 1 (NQO1), and glyceraldehyde-3phosphate dehydrogenase (GAPDH) were provided by Shanghai Sangon Biotech Co., Ltd. (Shanghai, China). TRIzol Reagent was purchased from Invitrogen (CA, USA). ChamQ SYBR qPCR Master Mix was provided by Vazyme Biotech Co., Ltd. (Nanjing, China). Except as otherwise expressly stated, other chemicals and reagents were used in analytical grade for meeting the needs of the study.

2.2. Preparation of DOP from D. officinale. Polysaccharides isolated from $D$. officinale (DOP) were prepared as previously described [23, 24]. First, D. officinale (100g) was crushed and extracted thrice with distilled water $(1: 20$, wt/ wt) for $2 \mathrm{~h}$. The combined extracts were filtered to remove impurities. Then, the supernatant was concentrated and deproteinized by adding the Sevag reagent. The alcohol concentration of the obtained liquid was adjusted to be $80 \%$ using edible alcohol. Afterward, the liquid was precipitated at $4^{\circ} \mathrm{C}$ overnight. The precipitate was collected by centrifuging at $3000 \mathrm{rpm}$ for $10 \mathrm{~min}$ at $4^{\circ} \mathrm{C}$ and redissolved in appropriate amounts of distilled water. Finally, the dissolved liquid was lyophilized using the vacuum freeze dryer (FreeZone Labconco, MO, USA) to obtain the DOP dry powder. The yield of polysaccharides in DOP was calculated as follows: polysaccharide yield $=($ polysaccharide weight $(\mathrm{g}) / \mathrm{Den}$ drobium of ficinale weight $(\mathrm{g})) \times 100 \%$.

The total amount of soluble carbohydrates in DOP was measured by the anthrone-sulfuric acid method [25]. First, $2 \mathrm{~mL}$ of DOP solution $(0.04 \mathrm{mg} / \mathrm{mL})$ was mixed with $6 \mathrm{~mL}$ of sulfuric anthrone $(1 \mathrm{mg} / \mathrm{mL})$ and heated at $90^{\circ} \mathrm{C}$ for $15 \mathrm{~min}$ followed by cooling to room temperature. The absorbance of the reaction liquid was measured using Multiskan Spectrum (Thermo, Finland) at $625 \mathrm{~nm}$. Different concentrations of D-glucose $(2,4,6,8,10$, and $12 \mathrm{mg} / 100 \mathrm{~mL})$ were 
prepared to draw the standard curve according to the aforementioned method.

\subsection{Determination of the Molecular Weight of DOP with} High-Performance Gel Permeation Chromatography. The molecular weights (MWs) of DOP were evaluated by highperformance gel permeation chromatography (HPGPC) according to the literature [26]. The HPGPC system was equipped with a 1525 HPLC instrument (Waters, MA, USA), a 2414 refractive index detector (Waters), and a 717 plus automatic sampler (Waters), which were acted on TSK G-5000 $0_{\text {PWXL }}\left(7.8 \times 300 \mathrm{~mm}^{2}\right.$, Tosoh Co., Ltd, Japan $)$ and TSK G- $3000_{\text {PWXL }}\left(7.8 \times 300 \mathrm{~mm}^{2}\right.$, Tosoh Co., Ltd $)$ columns in series. Then, $5 \mathrm{mg}$ of samples was dissolved in $2 \mathrm{~mL}$ of $0.02 \mathrm{M}$ potassium dihydrogen phosphate buffer solution and filtered through $0.45 \mu \mathrm{m}$ filters to obtain the supernatant. Further, $10 \mu \mathrm{L}$ of supernatant was injected into the HPGPC equipment for analysis. The analysis was performed with $0.02 \mathrm{M} \mathrm{KH}_{2} \mathrm{PO}_{4}$ at a flow rate of $0.6 \mathrm{~mL} / \mathrm{min}$ under the column and a detector temperature of $35^{\circ} \mathrm{C}$. The eight dextran standards of known MWs were used to calculate the MW (D5200, 11,600, 23,800, 48,600, 148,000, 273,000, 410,000, and 668,000$)$.

2.4. Animals and Treatment. Male ICR mice (6-8 weeks old) were purchased from Guangdong Medical Laboratory Animal Center (Foshan, China) and maintained with humidity of $50 \pm 10 \%$ at $23 \pm 2^{\circ} \mathrm{C}$ for a $12 \mathrm{~h} \mathrm{light/dark} \mathrm{cycle.} \mathrm{They}$ were given free access to food and water. All procedures were performed in accordance with the Regulations of Experimental Animal Administration issued by the Ministry of Science and Technology of the People's Republic of China. All animal experiments were approved by the Institutional Animal Care and Use Committee of Guangzhou University of Chinese Medicine.

All mice were randomly assigned to the following six groups ( $n=6$ /group): (1) control, (2) vehicle (APAP, $230 \mathrm{mg} / \mathrm{kg})$, (3) NAC (230 mg/kg APAP + $100 \mathrm{mg} / \mathrm{kg} \mathrm{NAC})$, (4) low dose of DOP $(230 \mathrm{mg} / \mathrm{kg}$ APAP $+50 \mathrm{mg} / \mathrm{kg}$ DOP $)$, (5) moderate dose of DOP $(230 \mathrm{mg} / \mathrm{kg}$ APAP $+100 \mathrm{mg} / \mathrm{kg}$ DOP), and (6) high dose of DOP $(230 \mathrm{mg} / \mathrm{kg}$ APAP $+200 \mathrm{mg} / \mathrm{kg}$ DOP). The mice from the control and vehicle groups were orally given distilled water. The other four groups were treated with corresponding doses of NAC and DOP orally for 30 days. All mice were intraperitoneally injected with APAP $4 \mathrm{~h}$ after the last treatment on day 30 , while the control group was injected with the same volume of normal saline. The blood of mice was collected from posterior orbital venous plexus $12 \mathrm{~h}$ after the APAP challenge. Then, the mice were sacrificed and liver tissues were collected followed by washing with normal saline three times. The blood and the liver tissues were used for biological and histological evaluation studies.

2.5. Histological Analysis. Liver tissues were fixed in $4 \%$ paraformaldehyde, embedded in paraffin, sectioned at $5 \mu \mathrm{m}$ thickness, and stained with hematoxylin and eosin (H\&E) following a standard protocol with minor modifications [27]. Histopathological changes in the liver were captured with an optical microscope at 200× magnification (E100, Nikon Corporation, Tokyo Japan).

2.6. Determination of ALT and AST Levels in Serum. The blood samples were kept at room temperature for $2 \mathrm{~h}$ and then centrifuged at $3000 \mathrm{rpm}$ for $10 \mathrm{~min}$ at $4^{\circ} \mathrm{C}$ to obtain serum. The ALT and AST levels in the serum were quantified using commercial kits (Nanjing Jiancheng Bioengineering Institute, Nanjing, China).

2.7. Determination of the GSH, MDA, CAT, T-AOC, and ROS Levels and the MPO Activity in Liver Tissues. The liver tissues were thawed and homogenized in the nine folds $(\mathrm{g} / \mathrm{mL})$ of ice-cold normal saline. The homogenate was centrifuged at $2500 \mathrm{rpm}$ for $10 \mathrm{~min}$ at $4^{\circ} \mathrm{C}$ to obtain the supernatant. The GSH, MDA, CAT, T-AOC, and ROS levels and MPO activity in the supernatant were measured using commercial kits (Nanjing Jiancheng Bioengineering Institute, Nanjing) and ELISA kit [28] according to the manufacturer's protocols.

2.8. Western Blot Analysis. Briefly, the liver tissues were homogenized using radioimmunoprecipitation assay (RIPA) buffer (R0020) (Solarbio, Beijing, China) containing phenylmethanesulfonyl fluoride (PMSF) (Beyotime, Shanghai, China). The cytosolic and nuclear proteins were extracted using a KeyGen nuclear kit and a cytoplasmic protein extraction kit (KeyGen Biotech, Jiangsu, China) according to the manufacturer's protocols, respectively. Protein concentration was quantified using a Bicinchoninic Acid Kit (BestBio, Shanghai, China). Equal amounts of protein samples were separated using sodium dodecyl sulfate-polyacrylamide gel electrophoresis and then transferred onto a polyvinylidene difluoride membrane. After being blocked in 5\% skimmed milk powder in Tris-buffered saline and Tween 20 (TBST) for $1 \mathrm{~h}$ at room temperature, the membranes were incubated with the following primary antibodies at $4^{\circ} \mathrm{C}$ overnight: Keap1 (1:200 dilution), Nrf2 (1:200 dilution), Histone H3 ( $1: 1000$ dilution), and $\beta$-actin ( $1: 1000$ dilution) followed by three washes of TBST. Then, the blots were incubated with horseradish peroxidase- (HRP-) conjugated secondary antibody $(1: 2000$ dilution) for $2 \mathrm{~h}$ at room temperature followed by three washes using TBST. Finally, the membranes were visualized using a Clarity Western Enhanced Chemiluminescence kit (Bio-Rad Laboratories Inc., CA, USA) and an automatic chemiluminescence image analysis system (Tanon Science \& Technology Co., Ltd., Shanghai, China).

2.9. Quantitative Real-Time PCR Analysis. Total RNA was extracted from liver tissues using TRIzol Reagent (Invitrogen, Life Technologies, CA, USA) according to the manufacturer's protocols. The purity of RNA was measured using Thermo Scientific NanoDrop 2000 (MA, USA). Next, the RNA was reverse transcribed to cDNA using a reverse transcriptase kit (TaKaRa Biotech, Kyoto, Japan). The quantitative real-time PCR (qRT-PCR) analysis was performed using a ChamQ SYBR qPCR Master Mix (Vazyme Biotech Co., Ltd., Nanjing, China) with the CFX Manager software (Bio-Rad Laboratories Inc.). GAPDH was analyzed in each sample to normalize expression. The primers used in this 
TABle 1: Primers used in this study.

\begin{tabular}{lcc}
\hline Primer name & Primer sequence $\left(5^{\prime}-3^{\prime}\right)$ & Product size \\
\hline GCLC forward & GCACATCTACCACGCAGTCAAGG & 273 \\
GCLC reverse & GCCTCCACAGTGTTGAACTCAGAC & 273 \\
GCLM forward & GCTTCGCCTCCGATTGAAGATGG & 380 \\
GCLM reverse & ACGATGACCGAGTACCTCAGCAG & 380 \\
NQO1 forward & TCACAGGTGAGCTGAAGGACTCG & 367 \\
NQO1 reverse & CGCAGGATGCCACTCTGAATCG & 367 \\
HO-1 forward & TCTGGTATGGGCCTCACTGG & 350 \\
HO-1 reverse & AATGTTGAGCAGGAAGGCGG & 350 \\
GAPDH forward & AATGGTGAAGGTCGGTGTGAACG & 235 \\
GAPDH reverse & TCGCTCCTGGAAGATGGTGATGG & 235 \\
\hline
\end{tabular}

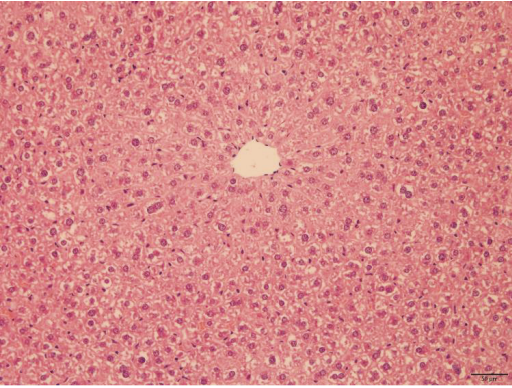

Control

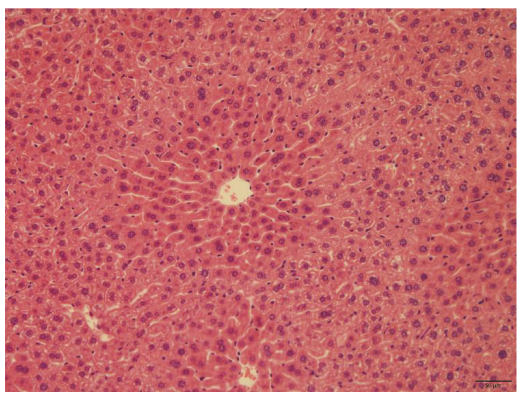

$\operatorname{DOP}(50 \mathrm{mg} / \mathrm{kg})$

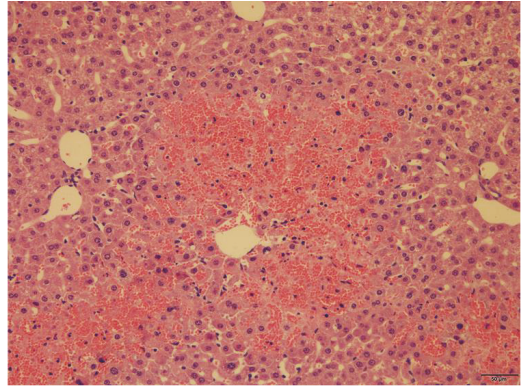

Vehicle

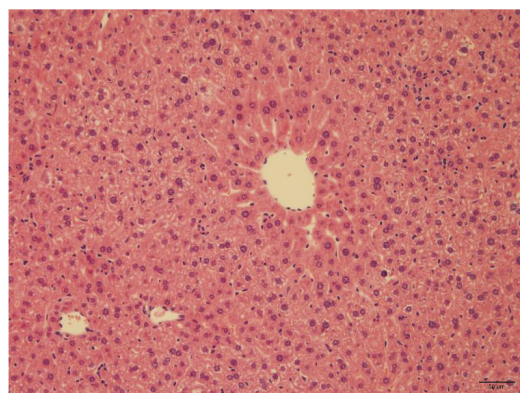

DOP $(100 \mathrm{mg} / \mathrm{kg})$

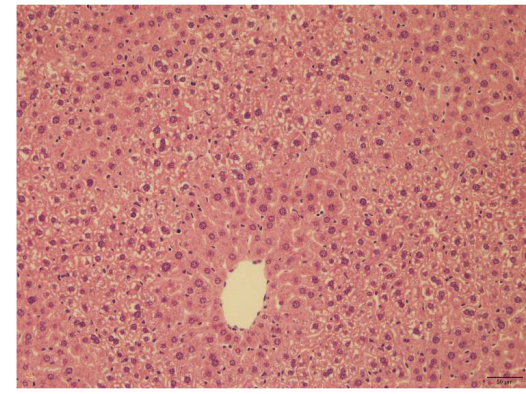

NAC

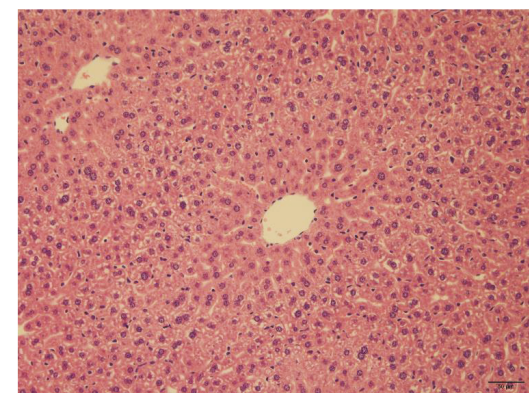

DOP $(200 \mathrm{mg} / \mathrm{kg})$

FIGURE 1: Results of histopathological examination of the mouse liver sections (H\&E staining, magnification 200x). Scale bar indicates $50 \mu \mathrm{m}$.

study are listed in Table 1 . The relative expression was analyzed by the $2^{-\Delta \Delta \mathrm{Ct}}$ method.

2.10. Statistical Analysis. All the experimental data were represented as means \pm standard error of the mean (SEM). One-way analysis of variance followed by the multiple comparisons of least significant difference test was used for all the data analysis. The analysis was performed using the Statistical Product and Service Solutions software 20.0 (SPSS Inc., NY, USA). A $P$ value $<0.05$ or $<0.01$ was considered as statistically significant.

\section{Results}

3.1. Determination of Carbohydrate Content in DOP. Dry polysaccharides $(5.15 \mathrm{~g})$ were obtained from $D$. officinale $(100 \mathrm{~g})$. The yield of DOP was $21.07 \%$. The carbohydrate content was determined based on the established linear curve of D-glucose $\left[Y=0.0054 X-0.0354\left(R^{2}=0.9992\right)\right]$. The content of total carbohydrate in DOP was $29.52 \pm 0.99 \%$.

3.2. Determination of $M W$ of DOP Using HPGPC. The MW of DOP was based on the calibration curve of dextran standards $\quad\left[\log \mathrm{MW}=-0.4891 \mathrm{~V}+13.975\left(R^{2}=0.9981\right)\right]$. The MW of DOP was found to be $8551 \mathrm{Da}$.

3.3. DOP Alleviated APAP-Induced Liver Injury. The mice were orally given DOP in advance to investigate the effects of DOP on APAP-induced hepatotoxicity. The histopathologic features of H\&E-stained liver sections are shown in Figure 1. Clear hepatic lobules and nuclei and no inflammation infiltration were observed in the control group. However, massive hepatic necroses, focal intrahepatic hemorrhage, and inflammation were noted around the 


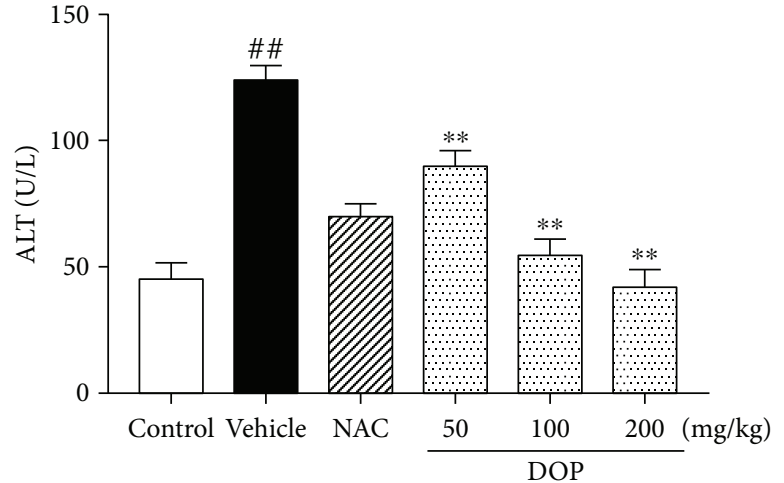

(a)

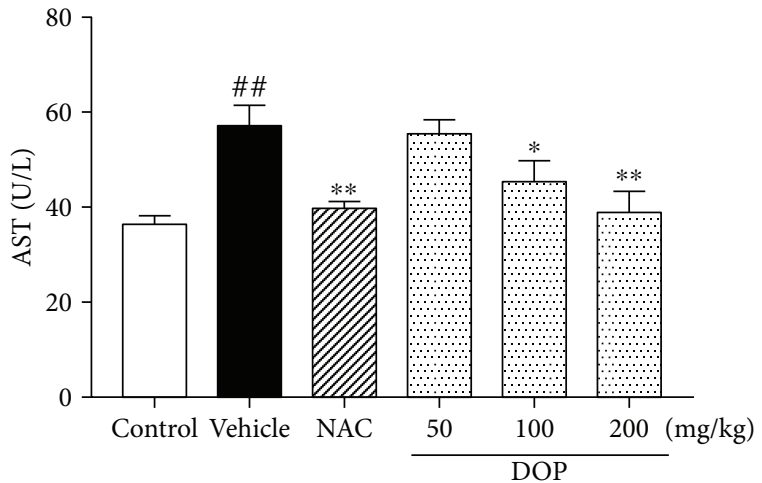

(b)

Figure 2: Effect of polysaccharides isolated from Dendrobium officinale (DOP) on the serum levels of alanine transaminase (ALT) (a) and aspartate aminotransferase (AST) (b) in mice. Data are presented as mean \pm SEM $(n=6) .{ }^{\# \#} P<0.01$ versus the control group. ${ }^{*} P<0.05$ and ${ }^{* *} P<0.01$ versus the vehicle group.

central venous lesions in APAP-treated mice. Meaningfully, DOP treatment significantly ameliorated APAPinduced hepatotoxicity.

\subsection{DOP Alleviated APAP-Induced Liver Enzyme} Dysfunction. As shown in Figure 2, the levels of ALT and AST significantly increased in the vehicle group following APAP challenge $(P<0.01)$ compared with the control group. However, DOP (100 and $200 \mathrm{mg} / \mathrm{kg}$ ) treatment significantly reversed these changes $(P<0.01, P<0.05)$. These data indicated that DOP alleviated APAP-induced liver enzyme dysfunction.

3.5. DOP Suppressed APAP-Induced Oxidative Stress. Previous studies showed that oxidative stress was closely associated with liver injury. As shown in Figure 3(a), the GSH level in the vehicle group was significantly lower than that in the control group $(P<0.01)$. Beside, NAC treatment significantly increased the GSH level compared with that in the vehicle group $(P<0.01)$. DOP treatment also significantly increased the GSH level in the liver compared with that in the vehicle control $(P<0.01)$. The GSH levels in the moderate and high doses of DOP groups were much higher than those in the NAC group.

As shown in Figures 3(b)-3(d), APAP stimulation significantly increased the levels of MDA and ROS and MPO activity in the liver compared with the control group (all, $P<0.01$ ). Compared with the vehicle group, the NAC group significantly reduced the levels of MDA and ROS and MPO activity (all, $P<0.01$ ). DOP treatment also reduced the productions of $\mathrm{MDA}$ and ROS and the activity of MPO (all, $P<0.05$ ). These results suggested that DOP strongly suppressed APAP-induced hepatic oxidative stress.

3.6. DOP Increased the Antioxidative Capacity against APAPInduced Oxidative Stress. As shown in Figures 4(a) and 4(b), the CAT and T-AOC levels in the vehicle group (APAP) were significantly lower than those in the control group (all, $P<0.01$ ), whereas NAC upregulated the levels of CAT and T-AOC (all, $P<0.01$ ). Interestingly, three doses of DOP increased the CAT level (all, $P<0.01$ ), and DOP (100 and $200 \mathrm{mg} / \mathrm{kg})$ increased the T-AOC level $(p<0.01)$ compared with the vehicle group. However, DOP $(50 \mathrm{mg} / \mathrm{kg})$ had no effect on liver T-AOC level. The data also indicated that DOP had a powerful antioxidative capacity against APAP-induced oxidative stress.

3.7. Effect of DOP on Nrf2 Nuclear Translocation. Nrf2 nuclear translocation and cytoplasmic Keap1 were determined using Western blot analysis to estimate whether DOP affected the Nrf2-Keap1 signaling pathway. As shown in Figures 5(a) and 5(b), APAP stimulation caused a decrease in Nrf2 level in nuclei in the mice compared with the control group $(75.1 \%, P<0.01)$. However, DOP significantly promoted the Nrf2 nuclear translocation compared with the APAP group. The change in level Nrf2 with DOP treatment was as follows: $50 \mathrm{mg} / \mathrm{kg}, 0.27$-fold increase $(P<0.01)$; $100 \mathrm{mg} / \mathrm{kg}, 0.06$-fold increase $(P<0.01)$; and $200 \mathrm{mg} / \mathrm{kg}$, 0.16 -fold increase $(P<0.01)$. Besides, APAP alone also caused an increase in cytoplasmic Keap1 $(112 \%, P<0.05)$. Nevertheless, the expression level of cytoplasmic Keap1 was significantly reduced with $200 \mathrm{mg} / \mathrm{kg}$ DOP treatment compared with the APAP group $(P<0.05)$. These results demonstrated that DOP enhanced the nuclear translocation of Nrf2.

3.8. Effect of DOP on the Expression of Nrf2 Target Gene in the Liver. Four indexes, including GCLC, GCLM, HO-1, and NQO1, in the liver samples were measured using qRTPCR to investigate the effect of DOP on Nrf2 target genes. As shown in Figure 6, APAP stimulation resulted in decreases in the mRNA expression levels of GCLC, GCLM, HO-1, and NQO1. Nevertheless, DOP treatment significantly upregulated the mRNA expression levels of GCLC, GCLM, HO-1, and NQO1 compared with the APAP group. Among three doses of DOP, $200 \mathrm{mg} / \mathrm{kg}$ DOP displayed the strongest promotion in the mRNA expression levels of GCLC (1152\%), GCLM (747\%), NQO1 (1180\%), and HO1 (229\%) (all, $P<0.01)$. 


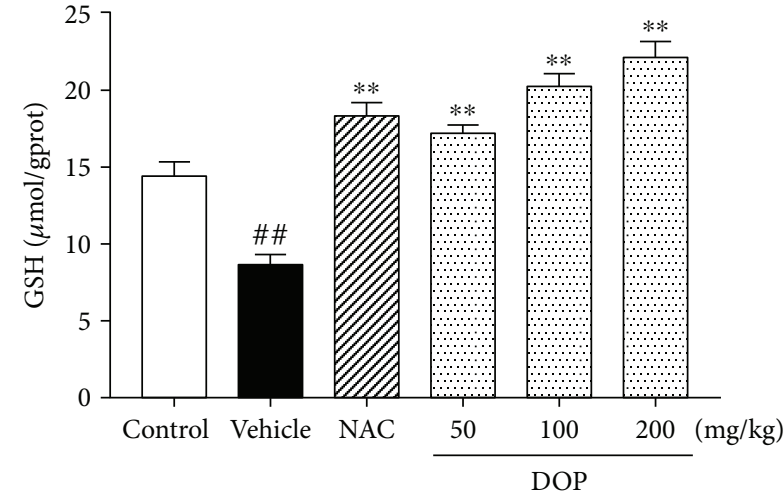

(a)

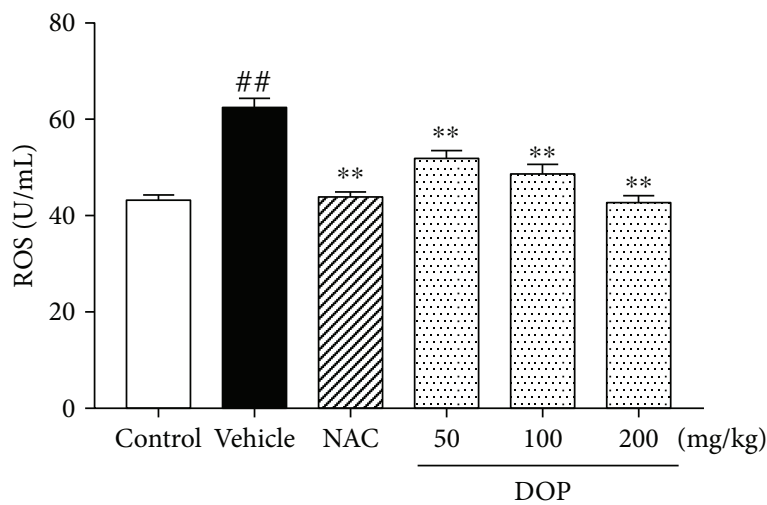

(c)

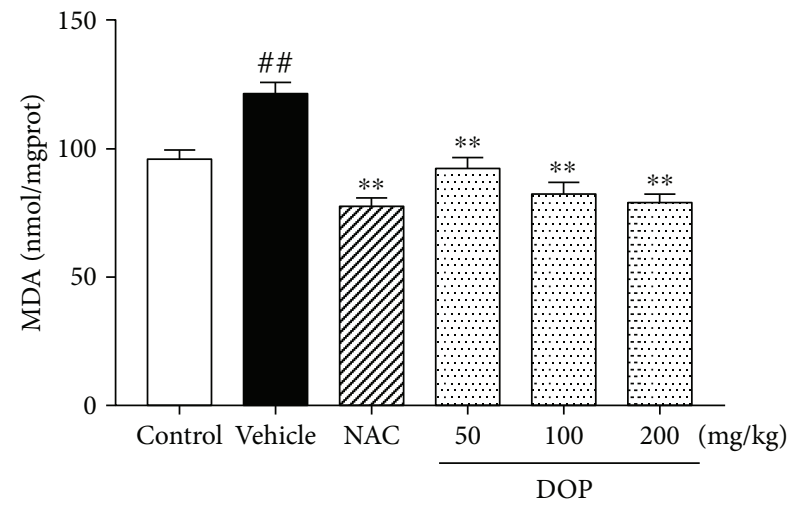

(b)

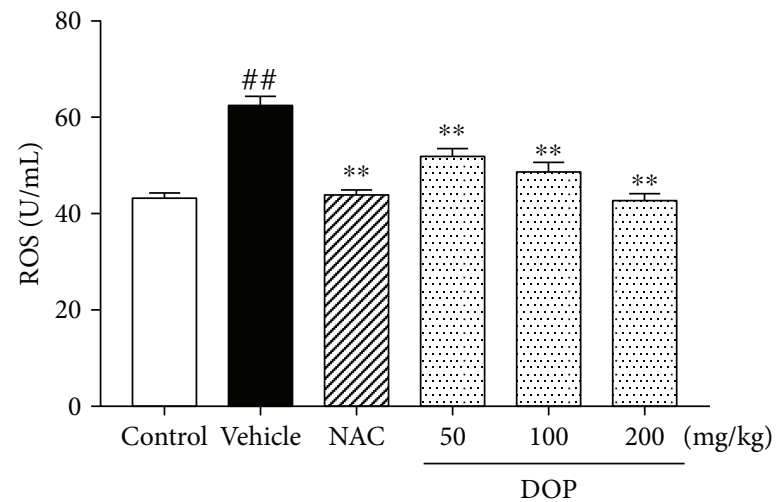

(d)

FIGURE 3: Effect of polysaccharides isolated from Dendrobium officinale (DOP) on glutathione (a), malondialdehyde (b), and reactive oxygen species (c) levels and myeloperoxidase activity $(\mathrm{d})$. Data are presented as mean $\pm \operatorname{SEM}(n=6) . \# \# P<0.01$ versus the control group. ${ }^{* *} P<0.01$ versus the vehicle group.

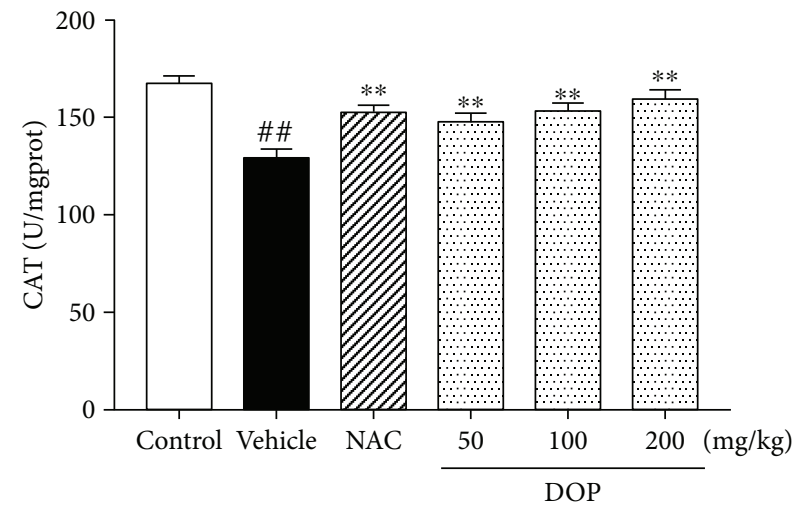

(a)

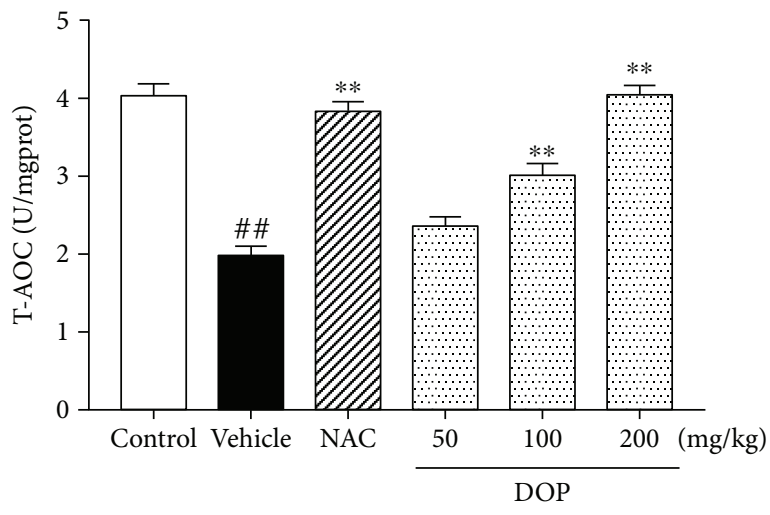

(b)

FIGURE 4: Effect of polysaccharides isolated from Dendrobium officinale (DOP) on catalase (a) and total antioxidant capacity (b). Data are

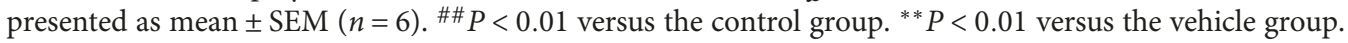

\section{Discussion}

The present study investigated the protective effects of DOP against APAP overdose-induced hepatotoxicity. The results indicated that DOP exerted the hepatoprotective effect by alleviating the oxidative stress through the Nrf2-Keap1 signaling pathway.
First, the dose of APAP was optimized. The study found that $230 \mathrm{mg} / \mathrm{kg}$ APAP was able to cause significant and stable hepatotoxicity in the preliminary experiment. Hence, $230 \mathrm{mg} / \mathrm{kg}$ APAP was used to induce liver injury in the following study. ALT and AST, two indicators of liver function, reflect the degree of liver injury [29]. Obviously, APAP administration significantly increased the levels of ALT and 


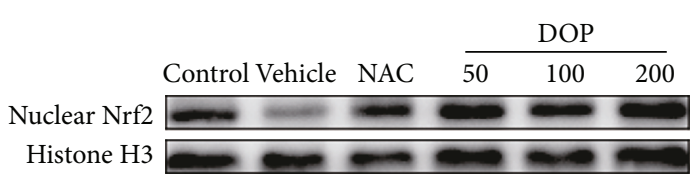

(a)

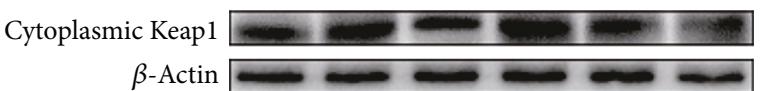

(c)

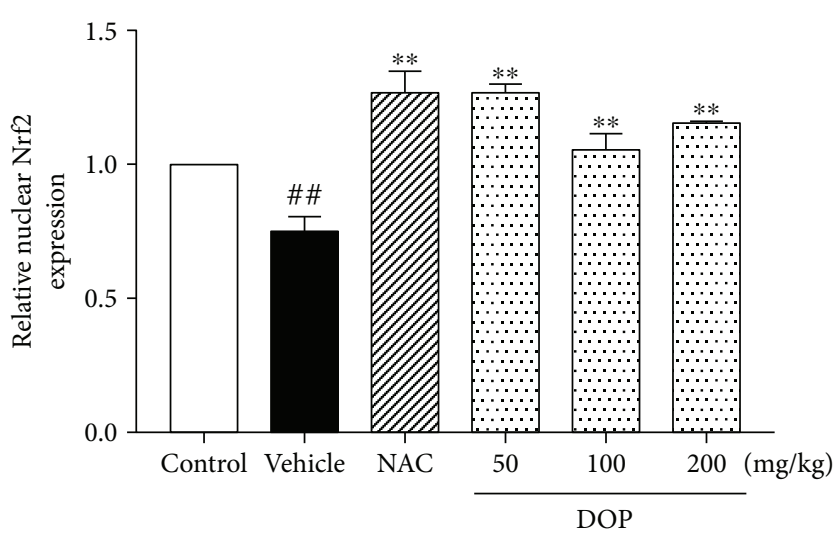

(b)

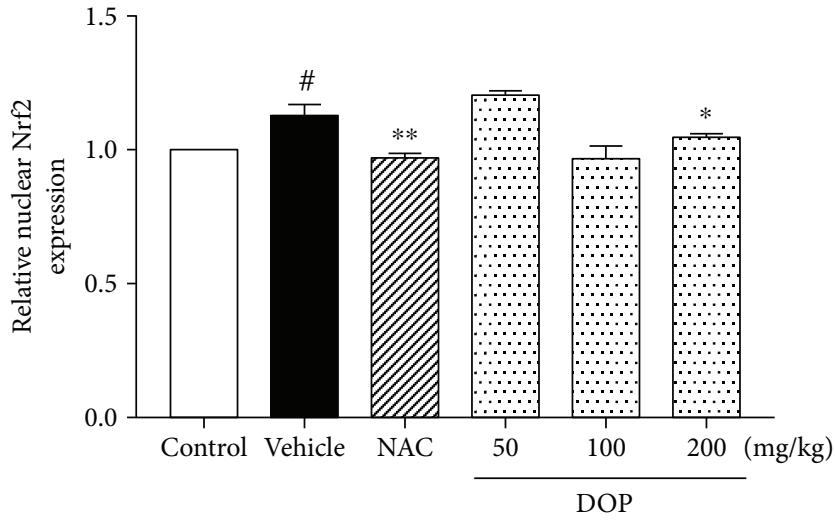

(d)

FIGURE 5: $(\mathrm{a}-\mathrm{c})$ Western blot analysis was used to measure nuclear Nrf2 and cytoplasmic Keap1 protein expression. Specific band intensities were quantified and normalized to histone $\mathrm{H} 3$ and $\beta$-actin, respectively (b-d). Data are presented as mean \pm SEM $(n=3)$. ${ }^{\#} P<0.05$ and \#\# $P<0.01$ versus the control group. ${ }^{*} P<0.05$ and ${ }^{* *} P<0.01$ versus the vehicle group.

AST (shown in Figure 2), whereas DOP treatment significantly inhibited the activities of ALT and AST in serum. Furthermore, histopathological analysis of liver tissues also revealed that DOP treatment reduced hepatic necroses and focal intrahepatic hemorrhage, besides arranging hepatic lobules in neat rows. The aforementioned results demonstrated that DOP exerted protective effects against APAP-induced liver injury.

An increasing body of evidence demonstrated an important role of oxidative stress in the liver injury induced by APAP [30-32]. Primarily, using cytochrome P450 enzymes, APAP-induced hepatotoxicity is ascribed to the formation of $N$-acetyl-p-benzoquinone imine (NAPQI), the toxic metabolite that causes hepatic glutathione (GSH) depletion and oxidative stress $[33,34]$. Afterward, residual NAPQI combines with mitochondrial proteins and causes mitochondrial dysfunction and ROS overproduction, finally resulting in DNA fragmentation and liver injury [35]. In addition, lipid peroxidation (LPO) has been a frequently invoked mechanism in ROS-induced cell death and liver injury [36]. Importantly, the massive LPO caused massive liver injury and acute liver failure within $4 \mathrm{~h}$ after APAP overdose [37]. Besides, GSH, ROS, MDA, CAT, and T-AOC levels are considered as the indicators of liver function [27, 38]. Lipid peroxidation was assessed by estimation of MDA in the liver tissues [39].

In the present study, APAP challenge significantly decreased the levels of GSH, CAT, and T-AOC in the mice compared with the control. Meanwhile, the production of ROS and MDA in the mice treated with APAP increased significantly. However, DOP treatment significantly upregulated the GSH, CAT, and T-AOC levels and downregulated the production of ROS and MDA, suggesting that DOP alleviated the hepatic oxidative stress induced by APAP. MPO activity is another marker of oxidative stress and inflammation [40]. The activity of MPO in the mice increased significantly after the APAP insult, leading to serious liver inflammation. However, DOP treatment significantly inhibited the activity of MPO. Combining these results, it was concluded that DOP treatment attenuated APAP-induced hepatic oxidative stress.

Next, the present study investigated the underlying mechanism of DOP against APAP-induced liver injury. Nrf2, a main regulator of the antioxidant defense system, mediates the antioxidant response element (ARE) [41]. On the contrary, Keap1 is a negative regulator of Nrf2 that combines with Cullin 3-based E3 ubiquitin ligase and results in the degradation of Nrf2 [42]. When activated, Nrf2 


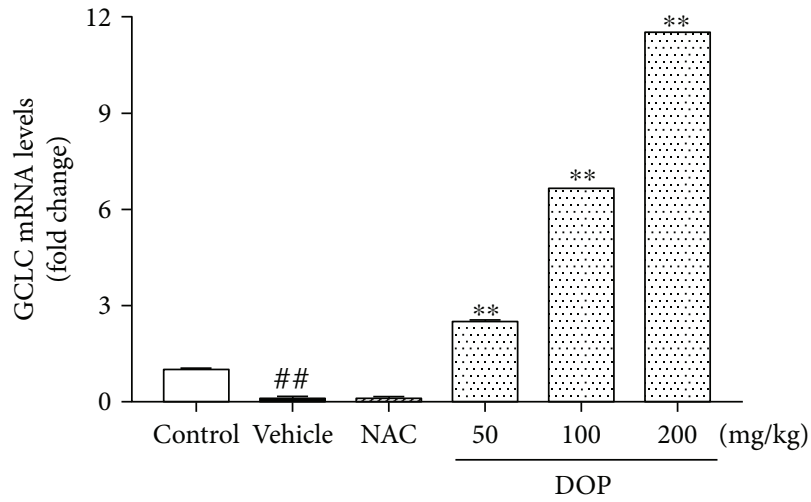

(a)

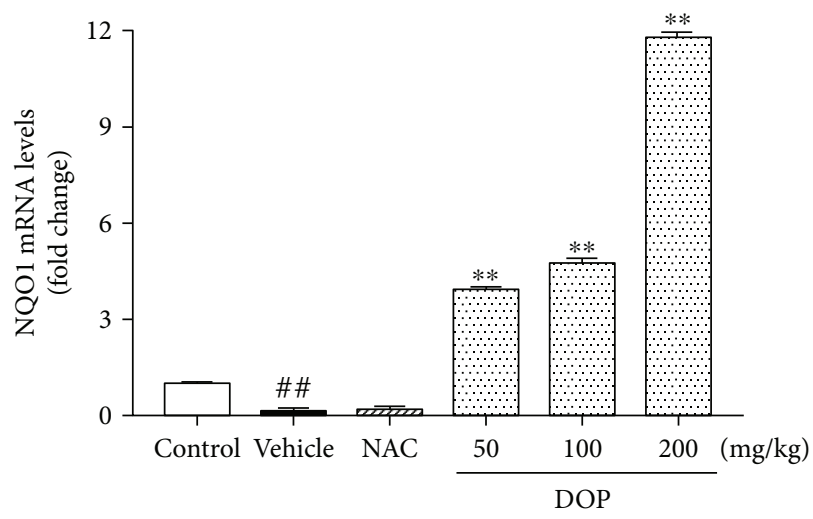

(c)

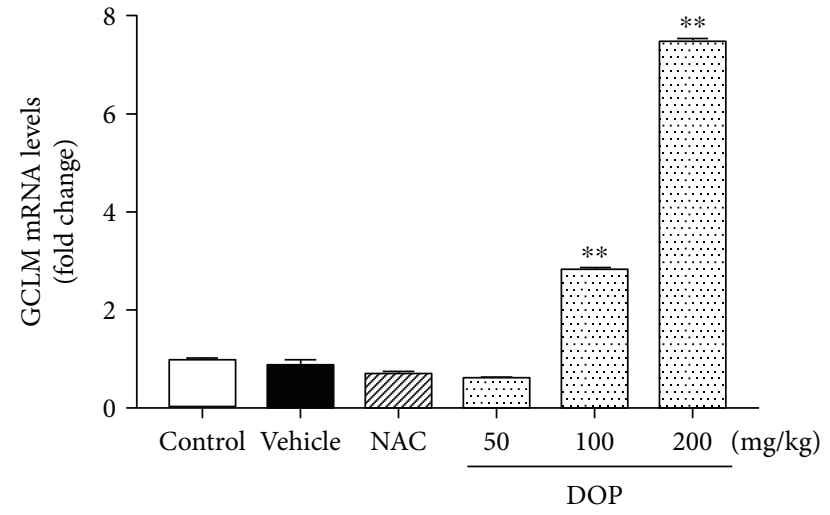

(b)

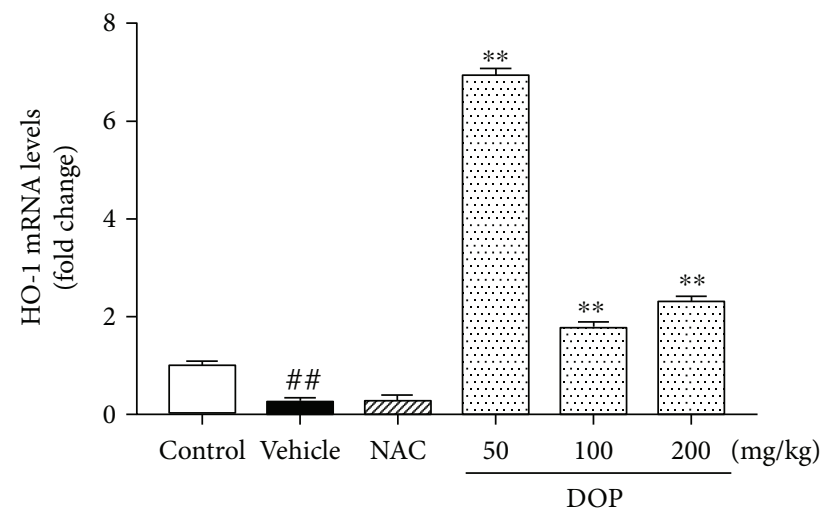

(d)

FIGURE 6: Effects of polysaccharides isolated from Dendrobium officinale (DOP) on the mRNA expression levels of GCLC (a), GCLM (b), NQO1 (c), and HO-1 (d). Total RNA from liver tissues was extracted and reverse transcribed into cDNA prior to quantitative real-time PCR analysis to detect mRNA expression of GCLC, GCLM, NQO1, and HO-1. Data are presented as mean \pm SEM $(n=3)$. \#\# $P<0.01$ versus the control group. ${ }^{* *} P<0.01$ versus the vehicle group.

translocates from the cytoplasm to the nucleus and regulates the expression of intracellular detoxifying and antioxidant genes, such as GCLC, GCLM, HO-1, and NQO1 $[43,44]$. Glutamate-cysteine ligase, consisting of GCLC and GCLM subunits, was reported to facilitate the synthesis of GSH [45]. NQO1 could reduce the NAPQI production and subsequently alleviate mitochondrial dysfunction caused by APAP [46]. HO-1 was demonstrated to promote the cleavage of heme to accelerate biliverdin formation and reduce intracellular ROS production [47]. Furthermore, the Nrf2-ARE signaling pathway was demonstrated to be involved in the hepatotoxicity induced by APAP [48]. Previous studies showed increased protein and mRNA expression levels of Nrf2 after effective drug treatment against APAP [41, 49, 50]. Importantly, the present study found that DOP treatment could increase the mRNA expression level of Nrf2 and also upregulate the expression of GCLC, GCLM, NQO1, and HO-1 compared with the APAP group. Moreover, DOP treatment could catalyze Nrf2 nuclear translocation and reduce the expression of Keap1, suggesting that DOP might exert a protective effect against APAP-induced hepatotoxicity via activating the Nrf2-Keap1 pathway.

\section{Conclusions}

This novel study demonstrated that DOP treatment exerted potentially hepatoprotective effects against APAP-induced liver injury. Further investigation of the underlying mechanisms indicated that DOP exerted the hepatoprotective effect by suppressing the oxidative stress and activating the Nrf2Keap1 signaling pathway. Collectively, DOP may be developed into a potential hepatoprotective drug, diet therapy, or functional food against APAP overdose-induced liver injury.

\section{Data Availability}

The data used to support the findings of this study are available from the corresponding author upon request.

\section{Conflicts of Interest}

The authors declare that there is no conflict of interest regarding the publication of this paper. 


\section{Authors' Contributions}

Guosheng Lin and Dandan Luo contributed equally to this work.

\section{Acknowledgments}

This work was supported by grants from Forestry Science and Technology Innovational Specific Project of Guangdong Province (nos. 2016KJCX006 and 2017KJCX007), National Key R\&D Program of China (no. 2017YFC0506200), Science and Technology Development Special Project of Guangdong Province (no. 2017A050506044), Guangdong Provincial Department of Education Feature Innovation Project (no. 2016KTSCX018), Science and Technology Project of Guangzhou (no. 201704030028), Science and Technology Planning Project of Guangdong Province Office of Education (no. 2014GKXM032), and Science and Technology Planning Project of Guangdong Province (no. 2013A022100001).

\section{References}

[1] W. M. Lee, "Drug-induced hepatotoxicity," New England Journal of Medicine, vol. 349, no. 5, pp. 474-485, 2003.

[2] R. J. Fontana, "Approaches to the study of drug-induced liver injury," Clinical Pharmacology \& Therapeutics, vol. 88, no. 3, pp. 416-419, 2010.

[3] C. Sgro, F. Clinard, K. Ouazir et al., "Incidence of druginduced hepatic injuries: a French population-based study," Hepatology, vol. 36, no. 2, pp. 451-455, 2002.

[4] A. Iorga, L. Dara, and N. Kaplowitz, "Drug-induced liver injury: cascade of events leading to cell death, apoptosis or necrosis," International Journal of Molecular Sciences, vol. 18, no. 5, 2017.

[5] L. Bell and N. Chalasani, "Epidemiology of idiosyncratic druginduced liver injury," Seminars in Liver Disease, vol. 29, no. 4, pp. 337-347, 2009.

[6] W. M. Lee, "Acetaminophen-related acute liver failure in the United States,” Hepatology Research, vol. 38, pp. S3-S8, 2008.

[7] A. M. Larson, J. Polson, R. J. Fontana et al., “Acetaminopheninduced acute liver failure: results of a United States multicenter, prospective study," Hepatology, vol. 42, no. 6, pp. 13641372, 2005.

[8] M. W. Russo, J. A. Galanko, R. Shrestha, M. W. Fried, and P. Watkins, "Liver transplantation for acute liver failure from drug induced liver injury in the United States," Liver Transplantation, vol. 10, no. 8, pp. 1018-1023, 2004.

[9] W. M. Lee, "Acetaminophen and the U.S. acute liver failure study group: lowering the risks of hepatic failure," Hepatology, vol. 40, no. 1, pp. 6-9, 2004.

[10] L. J. Chun, M. J. Tong, R. W. Busuttil, and J. R. Hiatt, "Acetaminophen hepatotoxicity and acute liver failure," Journal of Clinical Gastroenterology, vol. 43, no. 4, pp. 342-349, 2009.

[11] R. N. Jadeja, N. H. Urrunaga, S. Dash, S. Khurana, and N. K. Saxena, "Withaferin-A reduces acetaminophen-induced liver injury in mice," Biochemical Pharmacology, vol. 97, no. 1, pp. 122-132, 2015.

[12] L. Yip and R. C. Dart, "A 20-hour treatment for acute acetaminophen overdose," New England Journal of Medicine, vol. 348, no. 24, pp. 2471-2472, 2003.
[13] S. Bent, "Herbal medicine in the United States: review of efficacy, safety, and regulation - grand rounds at University of California, San Francisco Medical Center," Journal of General Internal Medicine, vol. 23, no. 6, pp. 854-859, 2008.

[14] R. Ahmad, S. P. Srivastava, R. Maurya, S. M. Rajendran, K. R. Arya, and A. K. Srivastava, "Mild antihyperglycaemic activity in Eclipta alba, Berberis aristata, Betula utilis, Cedrus deodara, Myristica fragrans and Terminalia chebula," Indian Journal of Science and Technology, vol. 1, no. 5, pp. 1-6, 2008.

[15] S. E. Kintzios, "Terrestrial plant-derived anticancer agents and plant species used in anticancer research," Critical Reviews in Plant Sciences, vol. 25, no. 2, pp. 79-113, 2006.

[16] D. K. Dash, V. C. Yeligar, S. S. Nayak et al., "Evaluation of hepatoprotective and antioxidant activity of Ichnocarpus frutescens (Linn.) R. Br. on paracetamol-induced hepatotoxicity in rats," Tropical Journal of Pharmaceutical Research, vol. 6, no. 3, pp. 755-765, 2007.

[17] J. Wu, J. F. Wang, L. Fang, H. L. Wu, M. W. Zhu, and R. W. Wang, "Domestic research progress of Dendrobium officinale Kimura et Migo," Chinese Pharmaceutical Journal, vol. 48, no. 19, pp. 1610-1613, 2013.

[18] T. B. Ng, J. Liu, J. H. Wong et al., "Review of research on Dendrobium, a prized folk medicine," Applied Microbiology and Biotechnology, vol. 93, no. 5, pp. 1795-1803, 2012.

[19] J. A. Teixeira da Silva and T. B. Ng, "The medicinal and pharmaceutical importance of Dendrobium species," Applied Microbiology and Biotechnology, vol. 101, no. 6, pp. 22272239, 2017.

[20] A. Lovegrove, C. H. Edwards, I. de Noni et al., "Role of polysaccharides in food, digestion, and health," Critical Reviews in Food Science and Nutrition, vol. 57, no. 2, pp. 237-253, 2017.

[21] C. C. Tian, X. Q. Zha, and J. P. Luo, “A polysaccharide from Dendrobium huoshanense prevents hepatic inflammatory response caused by carbon tetrachloride," Biotechnology \& Biotechnological Equipment, vol. 29, no. 1, pp. 132-138, 2015.

[22] X. Y. Wang, J. P. Luo, R. Chen, X. Q. Zha, and L. H. Pan, “Dendrobium huoshanense polysaccharide prevents ethanolinduced liver injury in mice by metabolomic analysis," International Journal of Biological Macromolecules, vol. 78, pp. 354-362, 2015.

[23] X. N. Zhao, J. L. Liang, H. B. Chen et al., "Anti-fatigue and antioxidant activity of the polysaccharides isolated from Millettiae speciosae Champ. Leguminosae," Nutrients, vol. 7, no. 10, pp. 8657-8669, 2015.

[24] D. Luo, C. Qu, G. Lin et al., "Character and laxative activity of polysaccharides isolated from Dendrobium officinale," Journal of Functional Foods, vol. 34, pp. 106-117, 2017.

[25] A. Piccolo, A. Zena, and P. Conte, "A comparison of acid hydrolyses for the determination of carbohydrate content in soils," Communications in Soil Science and Plant Analysis, vol. 27, no. 15-17, pp. 2909-2915, 1996.

[26] Y. F. Zheng, Q. Zhang, X. M. Liu, L. Ma, and F. Lai, "Extraction of polysaccharides and its antitumor activity on Magnolia kwangsiensis Figlar \& Noot," Carbohydrate Polymers, vol. 142, pp. 98-104, 2016.

[27] Q. Guo, Z. Shen, H. Yu et al., "Carnosic acid protects against acetaminophen-induced hepatotoxicity by potentiating Nrf2mediated antioxidant capacity in mice," The Korean Journal of Physiology \& Pharmacology, vol. 20, no. 1, pp. 15-23, 2016. 
[28] X. N. Wang, C. J. Zhang, H. L. Diao, and Y. Zhang, "Protective effects of curcumin against sodium arsenite-induced ovarian oxidative injury in a mouse model," Chinese Medical Journal, vol. 130, no. 9, pp. 1026-1032, 2017.

[29] J. Ozer, M. Ratner, M. Shaw, W. Bailey, and S. Schomaker, "The current state of serum biomarkers of hepatotoxicity," Toxicology, vol. 245, no. 3, pp. 194-205, 2008.

[30] H. Jaeschke, T. R. Knight, and M. L. Bajt, "The role of oxidant stress and reactive nitrogen species in acetaminophen hepatotoxicity," Toxicology Letters, vol. 144, no. 3, pp. 279-288, 2003.

[31] A. B. Reid, R. C. Kurten, S. McCullough, R. W. Brock, and J. A. Hinson, "Mechanisms of acetaminophen-induced hepatotoxicity: role of oxidative stress and mitochondrial permeability transition in freshly isolated mouse hepatocytes," Journal of Pharmacology and Experimental Therapeutics, vol. 312, no. 2, pp. 509-516, 2005.

[32] H. Jaeschke, M. R. McGill, and A. Ramachandran, "Oxidant stress, mitochondria, and cell death mechanisms in druginduced liver injury: lessons learned from acetaminophen hepatotoxicity," Drug Metabolism Reviews, vol. 44, no. 1, pp. 88106, 2012.

[33] D. C. Dahlin, G. T. Miwa, A. Y. Lu, and S. D. Nelson, "N-Acetyl-p-benzoquinone imine: a cytochrome P-450-mediated oxidation product of acetaminophen," Proceedings of the National Academy of Sciences of the United States of America, vol. 81, no. 5, pp. 1327-1331, 1984.

[34] L. P. James, P. R. Mayeux, and J. A. Hinson, "Acetaminopheninduced hepatotoxicity," Drug Metabolism and Disposition, vol. 31, no. 12, pp. 1499-1506, 2003.

[35] M. R. McGill, M. R. Sharpe, C. D. Williams, M. Taha, S. C. Curry, and H. Jaeschke, "The mechanism underlying acetaminophen-induced hepatotoxicity in humans and mice involves mitochondrial damage and nuclear DNA fragmentation," Journal of Clinical Investigation, vol. 122, no. 4, pp. 1574-1583, 2012.

[36] A. Negre-Salvayre, N. Auge, V. Ayala et al., "Pathological aspects of lipid peroxidation," Free Radical Research, vol. 44, no. 10, pp. 1125-1171, 2010.

[37] K. Du, A. Ramachandran, and H. Jaeschke, "Oxidative stress during acetaminophen hepatotoxicity: sources, pathophysiological role and therapeutic potential," Redox Biology, vol. 10, pp. 148-156, 2016.

[38] R. Jia, L. P. Cao, J. L. du et al., "Effects of carbon tetrachloride on oxidative stress, inflammatory response and hepatocyte apoptosis in common carp (Cyprinus carpio)," Aquatic Toxicology, vol. 152, pp. 11-19, 2014.

[39] P. Cao, J. Sun, M. A. Sullivan et al., "Angelica sinensis polysaccharide protects against acetaminophen-induced acute liver injury and cell death by suppressing oxidative stress and hepatic apoptosis in vivo and in vitro," International Journal of Biological Macromolecules, vol. 111, pp. 11331139, 2018.

[40] Y. Jing, K. Wu, J. Liu et al., “Aminotriazole alleviates acetaminophen poisoning via downregulating P450 2E1 and suppressing inflammation," PLoS One, vol. 10, no. 4, article e0122781, 2015.

[41] Y. M. Jiang, Y. Wang, H. S. Tan et al., "Schisandrol B protects against acetaminophen-induced acute hepatotoxicity in mice via activation of the NRF2/ARE signaling pathway," Acta Pharmacologica Sinica, vol. 37, no. 3, pp. 382-389, 2016.
[42] J. W. Kaspar, S. K. Niture, and A. K. Jaiswal, "Nrf2:INrf2 (Keap1) signaling in oxidative stress," Free Radical Biology and Medicine, vol. 47, no. 9, pp. 1304-1309, 2009.

[43] A. K. Jaiswal, "Nrf2 signaling in coordinated activation of antioxidant gene expression," Free Radical Biology and Medicine, vol. 36, no. 10, pp. 1199-1207, 2004.

[44] M. Kobayashi and M. Yamamoto, "Molecular mechanisms activating the Nrf2-Keap1 pathway of antioxidant gene regulation," Antioxidants \& Redox Signaling, vol. 7, no. 3-4, pp. 385394, 2005.

[45] O. W. Griffith, "Biologic and pharmacologic regulation of mammalian glutathione synthesis," Free Radical Biology and Medicine, vol. 27, no. 9-10, pp. 922-935, 1999.

[46] J. S. Moffit, L. M. Aleksunes, M. J. Kardas, A. L. Slitt, C. D. Klaassen, and J. E. Manautou, "Role of $\mathrm{NAD}(\mathrm{P}) \mathrm{H}$ : quinone oxidoreductase 1 in clofibrate-mediated hepatoprotection from acetaminophen," Toxicology, vol. 230, no. 2-3, pp. 197206, 2007.

[47] N. G. Abraham and A. Kappas, "Pharmacological and clinical aspects of heme oxygenase," Pharmacological Reviews, vol. 60, no. 1, pp. 79-127, 2008.

[48] A. Enomoto, K. Itoh, E. Nagayoshi et al., "High sensitivity of Nrf2 knockout mice to acetaminophen hepatotoxicity associated with decreased expression of ARE-regulated drug metabolizing enzymes and antioxidant genes," Toxicological Sciences, vol. 59, no. 1, pp. 169-177, 2001.

[49] C. Pang, Z. Zheng, L. Shi et al., "Caffeic acid prevents acetaminophen-induced liver injury by activating the Keap1-Nrf2 antioxidative defense system," Free Radical Biology \& Medicine, vol. 91, pp. 236-246, 2016.

[50] W. Wang, C. Guan, X. Sun et al., "Tanshinone IIA protects against acetaminophen-induced hepatotoxicity via activating the Nrf2 pathway," Phytomedicine, vol. 23, no. 6, pp. 589$596,2016$. 


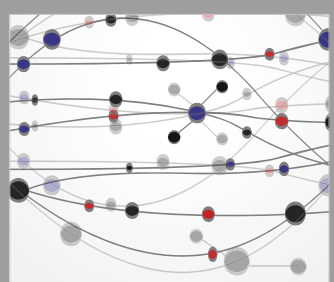

The Scientific World Journal
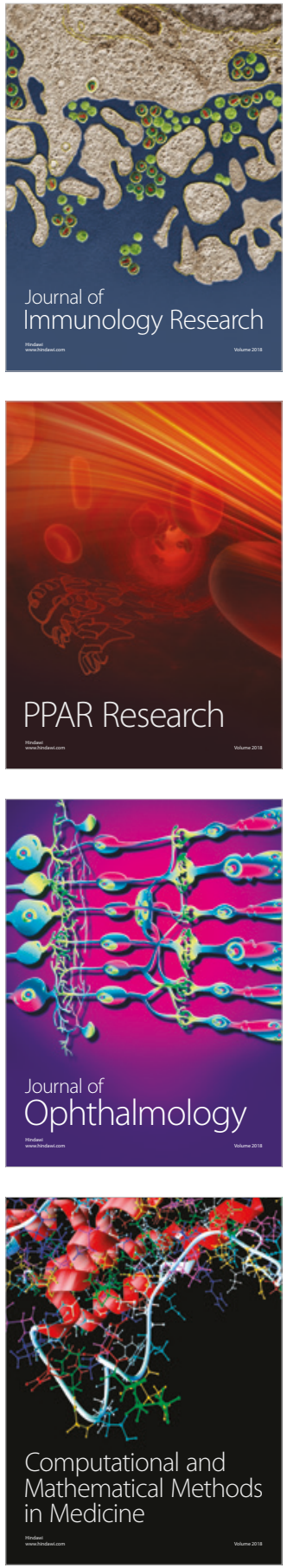

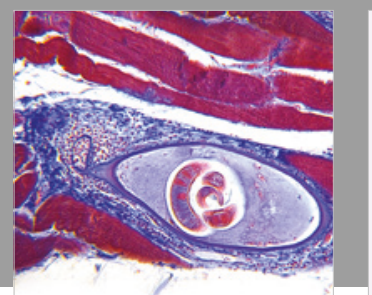

Gastroenterology Research and Practice

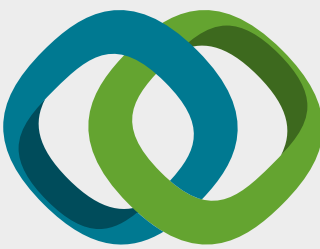

\section{Hindawi}

Submit your manuscripts at

www.hindawi.com
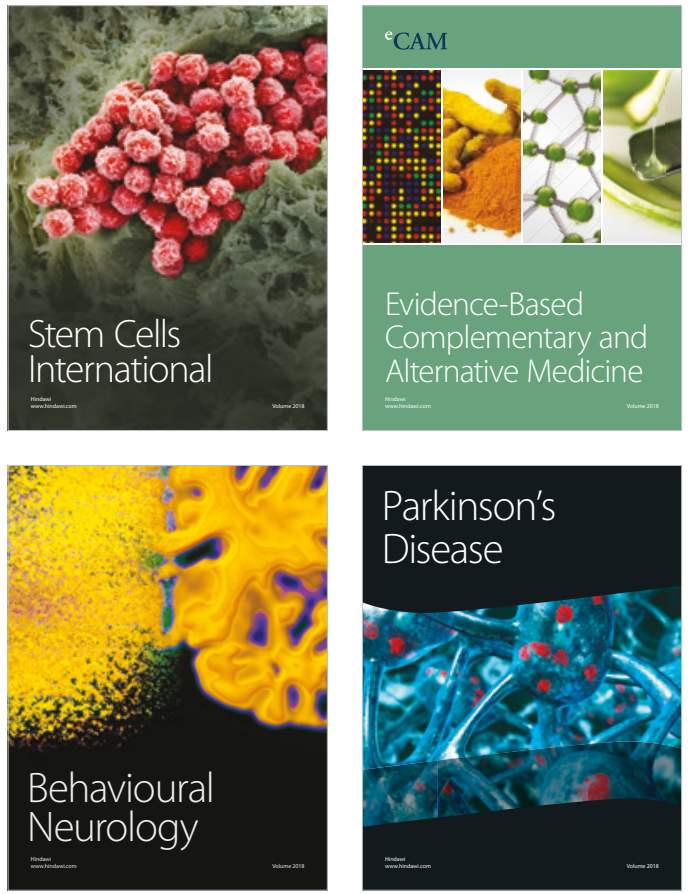

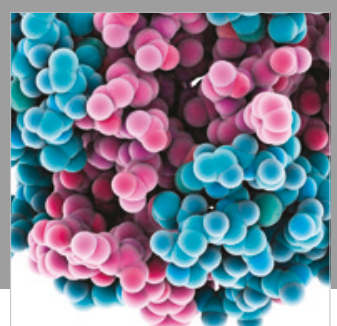

ournal of

Diabetes Research

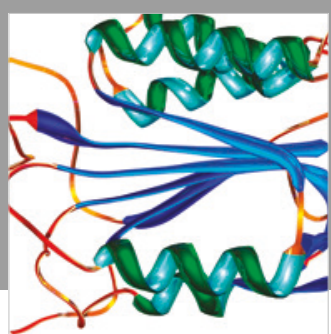

Disease Markers
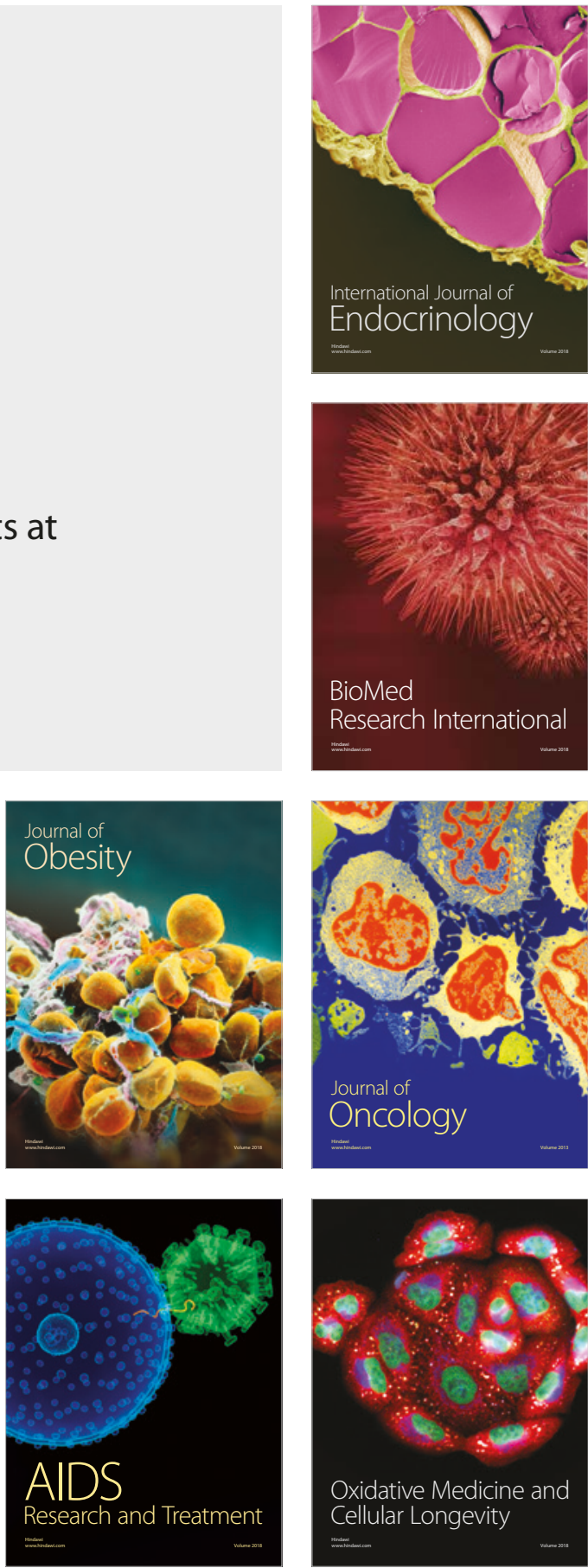